\title{
Article
}

\section{Single Maximal versus Combination Punch Kinematics}

Piorkowski, Barry Andrew, Lees, Adrian and Barton, Gabor J.

Available at http://clok.uclan.ac.uk/3757/

Piorkowski, Barry Andrew, Lees, Adrian and Barton, Gabor J. (2011) Single

Maximal versus Combination Punch Kinematics. Sports Biomechanics, 10 (1). pp. 1-11. ISSN 1476-3141

It is advisable to refer to the publisher's version if you intend to cite from the work. http://dx.doi.org/10.1080/14763141.2010.547590

For more information about UCLan's research in this area go to

http://www.uclan.ac.uk/researchgroups/ and search for < name of research Group>.

For information about Research generally at UCLan please go to http://www.uclan.ac.uk/research/

All outputs in CLoK are protected by Intellectual Property Rights law, including Copyright law. Copyright, IPR and Moral Rights for the works on this site are retained by the individual authors and/or other copyright owners. Terms and conditions for use of this material are defined in the policies page.

\section{CLoK}

Central Lancashire online Knowledge www.clok.uclan.ac.uk

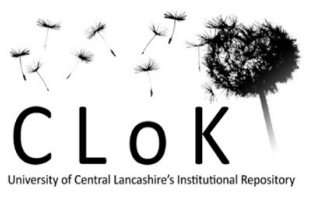




\section{Single maximal versus combination punch kinematics}

Barry A. Piorkowski, Adrian Lees and Gabor J. Barton

Research Institute for Sport and Exercise Sciences, Tom Reilly Building, Liverpool John Moores University, Byrom Street, Liverpool L3 3AF, United Kingdom

*email: a.lees@ljmu.ac.uk

tel: $+44(0) 1512314322$

fax: $+44(0) 1519046284$

Keywords: martial arts, boxing, punch, combination, kinematics 


\section{ABSTRACT}

The aim of this study was to determine the influence of punch type (Jab, Cross, Lead Hook and Reverse Hook) and punch modality (Single maximal, 'In-synch' and 'Out of synch' combination) on punch speed and delivery time. Ten competition-standard volunteers performed punches with markers placed on their anatomical landmarks for 3D motion capture with an eight-camera optoelectronic system. Speed and duration between key moments were computed. There were significant differences in contact speed between punch types $\left(F_{2,18,84.87}=105.76, P=0.001\right)$ with Lead and Reverse Hooks developing greater speed than Jab and Cross. There were significant differences in contact speed between punch modalities $\left(F_{2}, 64,102.87=\right.$ 23.52, $P=0.001$ ) with the Single maximal (mean $9.26 \pm 2.09 \mathrm{~m} / \mathrm{s}$ ) higher than 'Out of synch' (mean $7.49 \pm 2.32 \mathrm{~m} / \mathrm{s}$ ), 'In-synch' left (mean $8.01 \pm 2.35 \mathrm{~m} / \mathrm{s}$ ) or right lead (mean $7.97 \pm 2.53 \mathrm{~m} / \mathrm{s}$ ). Delivery times were significantly lower for Jab and Cross than Hook. Times were significantly lower 'In-synch' than a Single maximal or 'Out of synch' combination mode. Concluded is that a defender may have more evasiontime than previously reported. This research could be of use to performers and coaches when considering training preparations.

\section{INTRODUCTION}

The three main skills in combative sport are striking, wrestling and evasion. Striking uses body parts as weapons to cause damage to an opponent. Where both striking and wrestling are allowed, punching with the fist to the head is the most common way of winning a contest (Buse, 2006). This means that boxing skills are at the core of most Martial Arts. Past research into punching in boxing has mainly focused on single punches with respect to its end-point (fist) speed and estimated impact force 
delivered to a target. Single maximal punch contact speed has been measured at 8.9 $\mathrm{m} / \mathrm{s}$ for one professional boxer (Atha, et al., 1985) and $8.16 \mathrm{~m} / \mathrm{s}$ for amateurs (Walilko, et al., 2005). On both occasions fist displacement during punching was tracked from 2D optical camera images with a 3D accelerometer inserted into the glove of the boxer. This acceleration data was then mathematically integrated to confirm the velocity computed from the motion data (Atha, et al., 1985; Walilko, et al., 2005). Whiting et al. (1988), using 3D optical motion analysis, reported that combination punches developed speeds of only $5.9 \mathrm{~m} / \mathrm{s}$. These findings suggest that performers are unable to produce as much contact speed when throwing more than one punch in succession when compared to a Single maximal punch. This difference is also seen when comparing punch force. Impact force for a Single maximal boxing punch by elite boxers ranged between $3500-4800 \mathrm{~N}$ (Atha, et al., 1985; Smith, et al., 2000; Walilko, et al., 2005). Punches in competition (in the ring) or simulated combination punches (in the laboratory) produce around half the impact force of Single maximal punches (Pierce, et al., 2006; Smith, 2006; Stojsih, et al., 2008). Although these results are limited they do indicate that not all punch movements are executed in the same way with the same intent.

The kinematic comparisons between different types of punches and between Single maximal and combination punches are limited and this raises the question as to how combination punching differs from Single maximal punching across common punch types. Analysis of the kinematic characteristics of punch types and punch combination modalities will help to inform coaches and performers when preparing for competition. Different punch types and punch combination modalities could be rated so it is known which of them reach their intended target in the shortest time and also which generate the greatest contact speed. 
Therefore, the aim of this study was to determine the kinematic characteristics of boxing punches in order to identify the influence of punch type (Jab, Cross, Lead Hook and Reverse Hook) and punch modality (Single maximal, 'In-synch' combination and 'Out of synch' combination with left and right hand leads) on performance in terms of punch speed and duration.

\section{METHODS}

\section{Subjects}

Ten orthodox-stance boxers (mean $\pm \mathrm{s}$ :- age $24 \pm 4.4$ years, mass $72.5 \pm 14.1 \mathrm{~kg}$ ) of which seven were male and three were female ranging from local to European competition standard volunteered for the study. All subjects gave informed consent to participate in the study that was approved by the Institutional Ethics Committee.

\section{Data collection}

A life size strike-dummy was used as a punch target. It was modified to increase the surface area of the 'head' and also simulate a 'jaw' area. It had a heavy base which was sprung to allow movement of the 'head' when it was hit allowing it to return to the starting position. A multidirectional piezo-tronic accelerometer (A/131/V tri-axial, DJ Birchall, Mildenhall, UK) was mounted on top of the dummy head on the central core of the strike target and the unfiltered data which was sampled at $480 \mathrm{~Hz}$ was used to indicate punch contact. A passive reflective marker was placed next to the accelerometer so motion cameras could detect movement which would act to confirm punch contact.

[Insert Figure 1 here] 


\section{[Insert Figure 2 here]}

Eighteen reflective markers were placed on the anatomical landmarks of the subject in such a way that six segments could be identified to track motion in three dimensional space with six degrees of freedom of six upper body segments. The marker locations (Figure 2) were c7, left acromion, right acromion, left upper arm lateral, left upper arm medial, left elbow lateral, left elbow medial, left forearm, left wrist, left glove lateral, left glove medial, right upper arm medial, right upper arm lateral, right elbow lateral, right elbow medial, right forearm, right wrist and sacrum. Ten additional markers were used for calibration and located on the left shoulder lateral, left shoulder medial, right shoulder lateral, right shoulder medial, right glove lateral, right glove medial, left hip, right hip, left illiac crest and right illiac crest. The segments defined were the left forearm, left upper arm, right forearm, right upper arm, trunk and pelvis. All other segments depicted in Figure 2 are for illustration purposes only using a combination of markers located on the lower limbs and virtual markers. The fist was considered as part of the forearm as a whole segment since wrist movement is restricted with wraps made from fabric worn under the gloves. The 3D position of each marker was acquired at $240 \mathrm{~Hz}$ from eight infrared ceiling mounted cameras (ProReflex MCU240 system, Qualisys Inc., Gothenburg, Sweden). They provided a calibrated measurement volume of approximately $2^{\star} 2^{\star} 2 \mathrm{~m}$ in size. Kinematic analysis was undertaken using Visual 3D (Version 3.91, C-Motion Inc., Rockville, USA). All marker data were low-pass filtered using a $4^{\text {th }}$ order Butterworth filter with cut-off frequency of $12 \mathrm{~Hz}$ selected on the basis of a residual analysis and a qualitative evaluation of the data. 
The ground reaction force (GRF) data were collected from the rear foot of the subject by an embedded force platform (model 9821B11, Kistler Instruments, Hampshire, UK) sampled at $960 \mathrm{~Hz}$. GRF data were low-pass filtered using a $4^{\text {th }}$ order Butterworth filter with cut-off frequency of $40 \mathrm{~Hz}$.

Subjects performed a self selected warm up for approximately fifteen minutes that included light jogging, flexibility exercises and shadow boxing. They were then allowed a further five minutes (or longer if needed) to become familiarised with punching the strike target. During this time the target was moved to a comfortable position for all punches made by the subject. Markers were attached to the appropriate locations and a standing calibration trial was recorded. The anatomical markers were removed leaving the 18 markers for the dynamic trials (for the upper body segments in the model). Subjects familiarised themselves for a further five minutes with the punch type techniques and combination modalities, while wearing the markers. Subjects were then instructed in their own time to punch the target anywhere on the 'head' with the required technique or combination. The motive of every strike was a knockout of the virtual opponent by punching to the 'head' of a dummy target within the rules of Boxing.

Four punch types were performed as a Single (s) maximal punch and in three combinations (c) with the lead (left, 1) and reverse (right, 2) hands. The two Straight punch types were:- Jab - a straight left handed punch from the orthodox-stance (left foot forward) position; Cross - a straight right handed punch from the orthodoxstance position; The two Hook punch types were Lead Hook - a sweeping left handed punch from the orthodox-stance position; and Reverse Hook - a sweeping right handed punch from the orthodox-stance position. Thus, a Single maximal 
punch thrown (in orthodox-stance) by the left hand was identified as s1 and right hand, s2. The combination modalities were either 'In-synch' or 'Out of synch'. The 'Out of synch' combination punch sequences thrown with the same hand left or right would be c1111 or c2222 respectively. The 'In-synch' combinations were thrown with alternate hands either starting a left lead which was termed c1212 or right lead, c2121.

There was approximately $30 \mathrm{~s}$ break between trials. The coach of the subject or the investigator watched the trial as it was performed. At least two good trials were recorded and one selected for further analysis. Video 2D footage was taken in the sagittal plane for playback post data-collection.

\section{Data analysis}

Key moments were determined as ACTION (the start of the counter-movement seen in the $\mathrm{GRF}_{z}$ data); PROJ (when the forearm begins to move in the direction of the target - for combination punches the PROJ event did not start until the impact of the previous punch); IMPACT (the highest value in the spike of voltage data read by the accelerometer); and CONTACT (point in the movement immediately before the accelerometer picked up the IMPACT). CONTACT was typically $20 \mathrm{~ms}$ prior to IMPACT. The end of the strike segment (left or right forearms) represented the gloved fist in the model. The following variables were computed (1) punch (fist endpoint) contact speed (vector velocity); (2) punch contact speed to normalised limb length; (3) 3D displacement fist punch trajectory; (4) punch time defined between ACTION and CONTACT; (5) combination sequence (punch and recovery) time from CONTACT of the $1^{\text {st }}$ punch to CONTACT of the $4^{\text {th }}$ punch in sequence; (6) peak 
elbow flexion/extension angular velocity from PROJ to CONTACT; and (7) peak shoulder abduction/adduction angular velocity from PROJ to CONTACT. The PROJ and CONTACT event labels were used to normalise data to 101 points for graphical display. Data were smoothed over the whole of the movement and so would have been smoothed over IMPACT which is a known issue. While solutions do exist to overcome this, these are complex and were not available to us at the time of analysis. As all data were treated in the same way conditions could still be compared. Pre-projection data was not reported because the degree of variability in data was too great. Shoulder flexion was not reported since this was seen to take place prior to the projection phase. Either the second or third punch in the four punch combination was extracted from the motion data taking into consideration if a lead or reverse punch was required for analysis.

\section{Statistical analysis}

Shapiro-Wilk test was used to test for normality, an acceptable value for skew and kurtosis was decided at $< \pm 2.0$ (Vincent, 2005). If data were normally distributed within groups, a one-way repeated measures ANOVA was used. Homogeneity of variances of differences was taken into consideration. If Mauchly's Test of Sphericity reported Greenhouse-Geisser epsilon greater than 0.75 then the Huynh-Feldt corrected value was used. If less than 0.75 then the Greenhouse-Geisser corrected value was used (Girden, 1992). Post-hoc analysis to test for individual differences was a paired t-test with a Bonferroni correction. If the data were not normally distributed then a Mann-Whitney $U$ test was used for each pair of data sets. Pearson's $r$ two-tailed correlation analysis was used to investigate linear 
relationships between variables. All statistical tests were run using the computer statistical package SPSS, Chicago, USA with a level of significance set as $P<0.05$.

\section{RESULTS}

A typical 3D fist trajectory for a Single maximal punch of each type is given in Figure 3. Jab and Cross punch displacement was mostly along the anterior-posterior ( $Y$ ) axis moving forward in a straight line. Reverse Hook punches used a curved swinging motion with lateral movement along the transverse $(X)$ axis. At the beginning of the punch the Jab, Cross and Reverse Hook punches were all seen to drop down the longitudinal $(Z)$ axis, with the Reverse Hook dropping the most. There was a distinct period of movement for the Single maximal punch prior to projection where the fist seemed to move out of guard position and then back toward the target. The fist trajectories for combination punches, although not presented in the paper, are very similar to that of the Single maximal punch. The differences being that there was less of a drop along the longitudinal $(Z)$ axis at the start of the punch and there was no visible counter-movement. It also appeared in a combination punch that the fists were spread wider out of guard position at the start of the movement.

$$
\begin{aligned}
& \text { [Insert Figure } 3 \text { here] } \\
& \text { [Insert Figure } 4 \text { here] }
\end{aligned}
$$

Single maximal punches were selected to illustrate the general differences between the fist, elbow, shoulder and hip velocity components during a Cross and Lead Hook punch (Figure 4). The negative fist velocities indicate a countermovement which was a greater for Hook punches (Figure 4D, 4E, 4F) than for Cross 
(Figure 4A, 4B, 4C) punches. For the Cross punch there is evidence to suggest a sequential body movement with the velocity of the shoulder, hip, elbow and then fist peaking in sequence while the magnitude of the component velocity was in reverse order being hip, shoulder, elbow then fist (Figure 4B).

Contact speeds for all punches and punch combinations were normally distributed $(P>0.05)$. There was a significant difference in contact speed between punch types for all punch modalities $\left(F_{2,18,84.87}=105.76, P=0.001\right)$. Post-hoc analysis revealed Hook punches contact speed (Table 1) was significantly higher (Table 1) in the Lead and Reverse Hook punches than the Jab and Cross punches. The Cross had significantly higher contact speed than the Jab. There was no significant difference between Lead and Reverse Hooks.

There was a significant difference in punch contact speed between punch

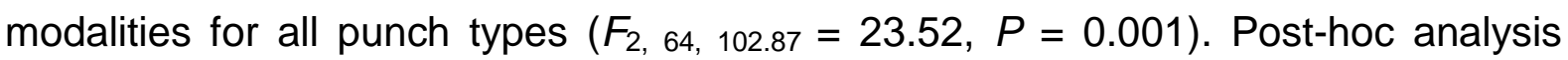
revealed Single maximal punch contact speed (Table 1) was significantly higher than 'Out of synch' combination punch contact speed, 'In-synch' left lead and 'In-synch' right lead combination punch contact speed (Table 1). There was no significant difference between combinations.

Table 1. Mean Punch Contact Speed (m/s) and Mean Punch Time (ms).

[Insert Table 1 here]

Delivery times (Table 1) were not normally distributed. With regard to punch type, they were significantly lower $(U=1929, Z=-4.34, P=0.001)$ for the Straight punches than for the Hook punches. However there was no significant difference in time between the Jab and Lead Hook (Table 1). With regard to punch modality, 
delivery times were shorter for the 'In-synch' combination punches than the Single maximal punch and 'Out of synch' combination punch modes (Table 1). There was a significant difference between Single maximal and 'Out of synch' combination modes $(U=326.5, Z=-4.56, P=0.001)$. There was a significant difference between Single maximal and 'In-synch' left lead combination $(U=222.5, Z=-5.56, P=0.001)$ and 'In-synch' right lead combination $(U=206, Z=-5.72, P=0.001)$. There was a significant difference between 'Out of synch' combination and 'In-synch' left lead combination $(U=439, Z=-3.48, P=0.001)$ and 'In-synch' right lead combination ( $U$ $=403.5, Z=-3.82, P=0.001)$. There was no significant difference in delivery times between leading with the right or left hand 'In-synch' (Table 1).

Combination sequence (punch and recovery) time from CONTACT of the $1^{\text {st }}$ punch to CONTACT of the $4^{\text {th }}$ punch was not normally distributed. The 'Out of synch' combination sequence time (mean $1.35 \pm 0.27 \mathrm{~s}$ ) was significantly higher than both 'In-synch' combinations leading with either the left $(U=458.9, Z=-3.29, P=0.001)$ or right hand $(U=416, Z=-3.70, P=0.001)$. There was no significant difference leading with the left (mean $1.06 \pm 0.46 \mathrm{~s}$ ) or right hand (mean $1.04 \pm 0.42 \mathrm{~s}$ ).

The role of the elbow was thought to be important for Straight punches and the shoulder for Hook punches. There was a significant positive correlation between normalised peak contact speed and peak elbow angular extension velocity for the Straight punches, $r_{80}=0.27, P=0.001$ (Figure 5). The correlation between normalised peak contact speed and peak shoulder angular abduction/adduction velocity for the Hook punch was not significant. 
[Insert Figure 5 here]

\section{DISCUSSION AND IMPLICATIONS}

The mean contact speed for all subjects for the Single maximal Cross $(8.22 \pm 1.08$ $\mathrm{m} / \mathrm{s}$ ) is comparable with previous studies which has been measured at $8.9 \mathrm{~m} / \mathrm{s}$ for one professional boxer (Atha, et al., 1985) and $8.16 \mathrm{~m} / \mathrm{s}$ for amateurs (Walilko, et al., 2005). Further, both Hook punches had significantly higher $(P<0.05)$ contact speed than the Straight punches confirming the findings of Whiting et al. (1988). The greater contact speed of the Hook punch is due to the different kinematics and punch trajectory between Straight and Hook punches. Straight punches move forward utilising elbow extension, while Hook punches sweep round and up relying on shoulder flexion and adduction providing a greater range of movement for a Hook punch compared to a Jab or Cross. The length of the acceleration path for a Hook punch can be longer than the Straight punch. This is seen in Figure 1 for the Single maximal Hook and is due to 'winding-up' in the counter-movement.

The suggestion that the greater acceleration path provides more time to generate greater endpoint speed (Whiting, et al., 1988) is only true for the Single maximal Reverse Hook. The Single maximal Lead Hook takes no longer to complete that the Jab and Cross. The greater punch contact speed of the Lead Hook is therefore not only dependent on the length of the acceleration path but must also be related to the acceleration of the arm and this is governed by the torque generated at the shoulder. This finding has implications for strength and conditioning training of shoulder muscles. It is not clear why the Reverse and Lead Hooks differ so markedly 
in their time for execution. Perhaps this is because in orthodox-stance (left foot forward) in guard position the boxer is side-on which means there may be more time needed to prepare for the Reverse Hook punch to improve stability of footwork rather than for punch speed. These ideas would be interesting for further study.

The results for all modalities show that although Hook punches generate greater contact speed than Straight punches they also take more time to reach their intended target. Past researchers have determined punch time from PROJ to CONTACT. Single maximal Cross punch time has been recorded at $100 \mathrm{~ms}$ with an additional 200 ms for the advance (Atha, et al., 1985). Whiting et al. (1988) recorded a mean combination Cross punch cycle time of $132 \pm 21 \mathrm{~ms}$ and Reverse Hook mean $143 \pm 24 \mathrm{~ms}$. The results were similar for this investigation with the mean Single maximal Cross punch time from PROJ to CONTACT being $223 \pm 99 \mathrm{~ms}$ and combination Reverse Hook mean $125 \pm 34 \mathrm{~ms}$. In this study however, punch time was considered to be from ACTION to CONTACT. Hook punch time (mean all modes $=477 \pm 203 \mathrm{~ms}$ ) from ACTION to CONTACT is longer than Straight punches (mean all modes $=357 \pm 178 \mathrm{~ms}$ ). A defending boxer that can spot early visual cues therefore has more time to evade punches than suggested by previous research (Atha, et al., 1985; Whiting, et al., 1988).

Single maximal punches generated the highest contact speed and were significantly higher $(P<0.05)$ than 'Out of synch' combination, 'In-synch' left lead and 'In-synch' right lead combinations. There is no past research in this area to compare results but a suggested reason why Single maximal punches generated more contact speed than combinations punches could be that there is more time spent in the counter-movement. Although this was not specifically quantified, the 
durations of the Single maximal punches (mean $607 \pm 221 \mathrm{~ms}$ ) were longer than the combination punches (mean $420 \pm 65 ; 321 \pm 166 ; 320 \pm 163 \mathrm{~ms}$ ) for the 'Out of synch', 'In-synch' left and right lead respectively. Qualitative analysis of the recorded video showed a knee flexion/extension counter-movement at the start of the Single maximal punch. This extra preparation time could be a reason why Single maximal punches have greater contact speed than combination punches. A second reason could be that counter-movements are widely associated with the stretch-shortening phenomena which is known to enhance performance. Combination punches have shorter punch times than Single maximal punches as time is saved by restricting the counter-movement.

There was no significant difference in punch contact speed between the combination modalities. In delivery time however, the 'Out of synch' combination punch took significantly longer $(P<0.05)$ to reach the target than the 'In-synch' combination punches. This result could be attributed to the performer having to spend more time recovering from the punch in order to prepare for the next punch with the same hand being in use. Punch combination flow is difficult to quantify but may be related to the time between punches in the sequence. Combination sequence (punch and recovery) time from CONTACT of the $1^{\text {st }}$ punch to CONTACT of the $4^{\text {th }}$ punch was calculated as an indicator of combination punch flow. From the results there is evidence to suggest that throwing punches in either a left then right or a right then left sequence flows with less time needed to move than using only the same hand to punch in combination as there was significant difference $(P<0.05)$ between 'In-synch' and 'Out of synch' combination sequence time. 
This study used a combination of highly skilled male and female boxers. While the speed of punches thrown by females generally is lower than males it is assumed that the technique used by female boxers is similar to that of male boxers. This is reasonable based on their skill level. These female boxers train and are coached in the same way as the male boxers. There was no indication that the techniques used differed with gender so in this study the data from male and female subjects was taken together. The findings and observations noted in the discussion enable comment to be made regarding preparation for boxing. Firstly, an ability to use early visual cues related to the counter-movement (e.g. knee flexion or the fist moving out of guard position) preceding a punch could give a combatant extra time to evade a strike. Secondly, knowledge of the trajectory of each punch type will enable a defending combatant to protect the head appropriately and deflect an incoming punch or move the head outside the line of the punch path. Straight punches can be slipped by moving the head laterally. Hook punches can be ducked by dipping the head under sweeping motion or blocking the punch with the same arm in a mirrored fashion. Thirdly, as there is more than one path of punch trajectory it would be advisable for boxers to develop isometric neck tension in all directions. Other strength training would be appropriate to enhance performance particularly shoulder strength to maximise the speed of Hook punches and elbow strength to maximise the speed of Straight punches.

\section{CONCLUSION}

The aim of this study was to determine the kinematic characteristics of boxing punches in order to identify the influence of punch type and punch modality on 
performance in terms of punch speed and duration. The results for punch contact speed of all subjects are comparable with previous studies. Hook punches had a higher contact speed than the Straight punches but Hook punch delivery time is slower than Straight punches. Single maximal punch modes generated higher contact speed than combination punch modes but combination punch modes have a shorter punch delivery time than Single maximal punch modes. From the results of this study a defending boxer may have more time to evade punches than previously reported in other work. This research could be of use to performers and coaches by informing punch attack and punch defence pre-fight training preparations. 


\section{REFEERENCES}

Atha, J., Yeadon, M. R., Sandover, J., \& Parsons, K. C. (1985). The damaging punch. British Medical Journal (Clin Res Ed), 291(6511), 1756-1757.

Buse, G. J. (2006). No holds barred sport fighting: a 10 year review of mixed martial arts competition. British Journal of Sports Medicine, 40(2), 169-172.

Girden, E. (1992). ANOVA: Thousand Oaks: Sage Publications.

Pierce, J., Reinbold, K., Lyngard, B., Goldman, R., \& Pastore, C. (2006). Direct Measurement of Punch Force During Six Professional Boxing Matches. Journal of Quantitative Analysis in Sports, 2(2), 1-17.

Smith, M. S. (2006). Physiological Profile of Senior and Junior England International Amateur Boxers. Journal of Sports Science and Medicine (CSSI), 74-89.

Smith, M. S., Dyson, R. J., Hale, T., \& Janaway, L. (2000). Development of a boxing dynamometer and its punch force discrimination efficacy. Journal of Sports Sciences, 18(6), 445-450.

Stojsih, S., Boitano, M., Wilhelm, M., \& Bir, C. (2008). A prospective study of punch biomechanics and cognitive function for amateur boxers. British Journal of Sports Medicine, published online 19 Nov 2008.

Vincent, W. (2005). Statistics in Kinesiology: Human Kinetics.

Walilko, T. J., Viano, D. C., \& Bir, C. A. (2005). Biomechanics of the head for Olympic boxer punches to the face. British Journal of Sports Medicine, 39(10), 710-719.

Whiting, W. C., Gregor, R. J., \& Finerman, G. A. (1988). Kinematic analysis of human upper extremity movements in boxing. American Journal of Sports Medicine, 16(2), 130-136. 


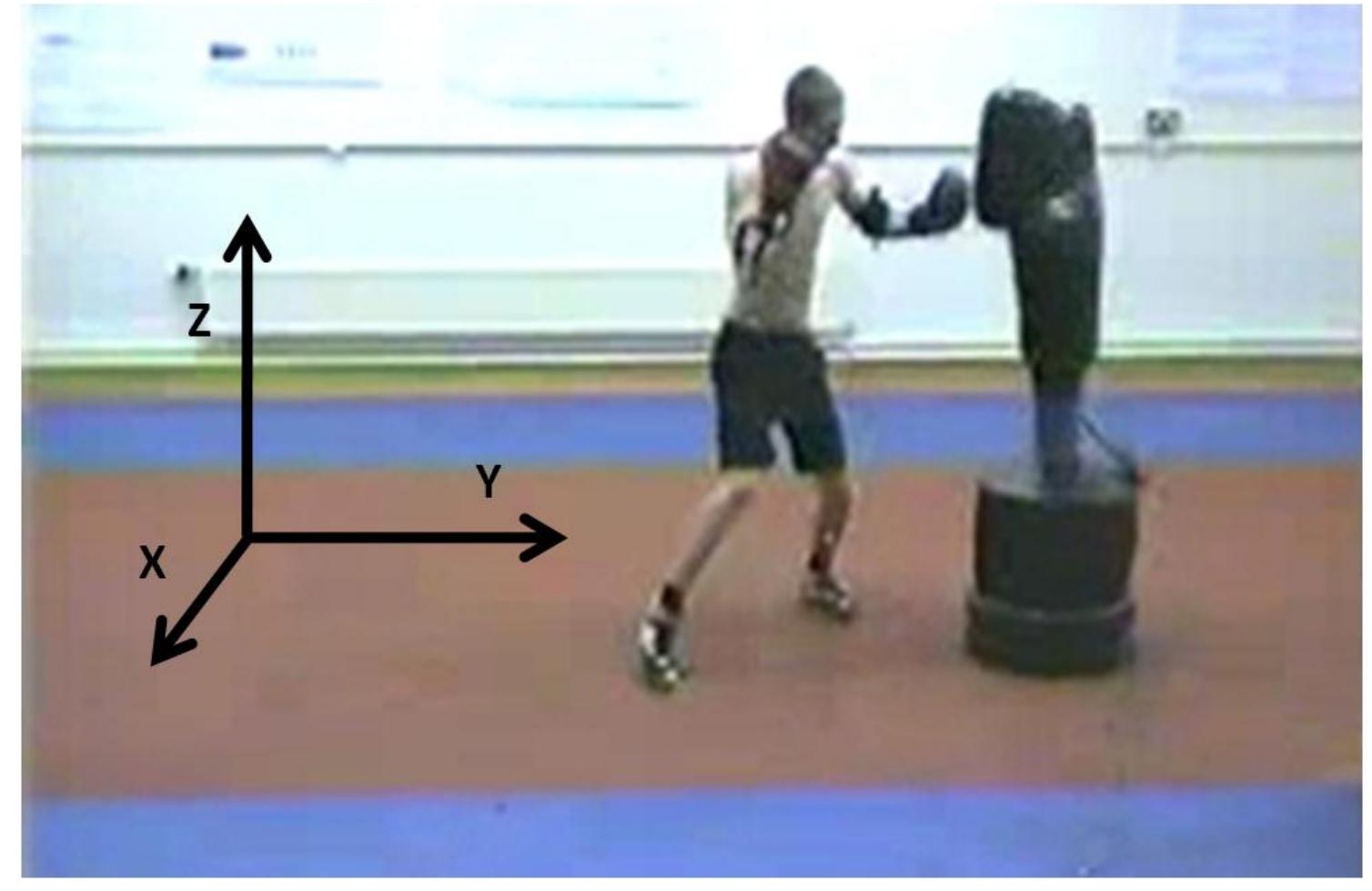

Figure 1. Lab Coordinate System and Dummy Target. 


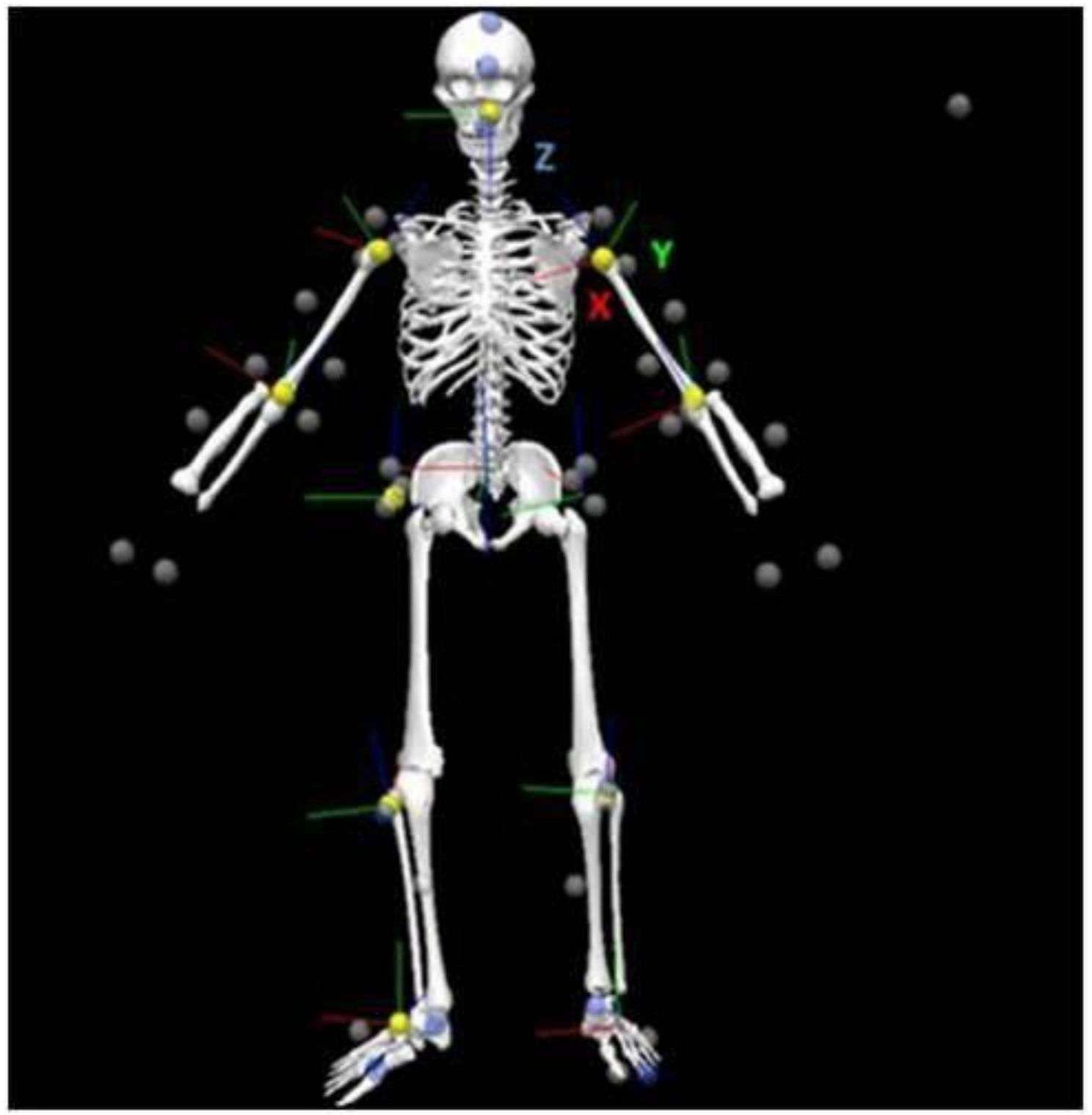

Figure 2. Local Coordinate System of the Model. 


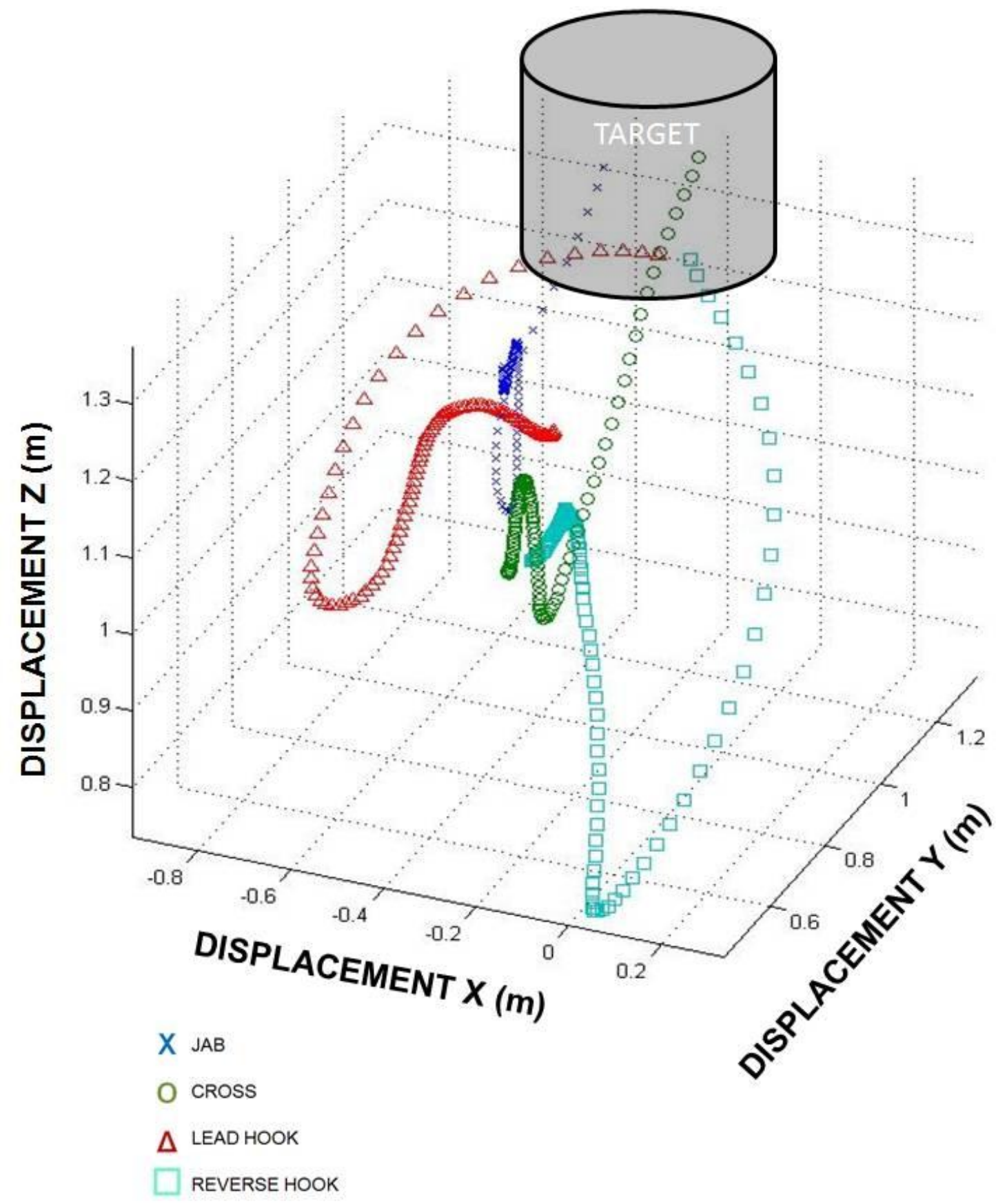

Figure 3. Typical 3D Displacement Graph of a Single maximal Punch. The target is for illustrative purposes only. 

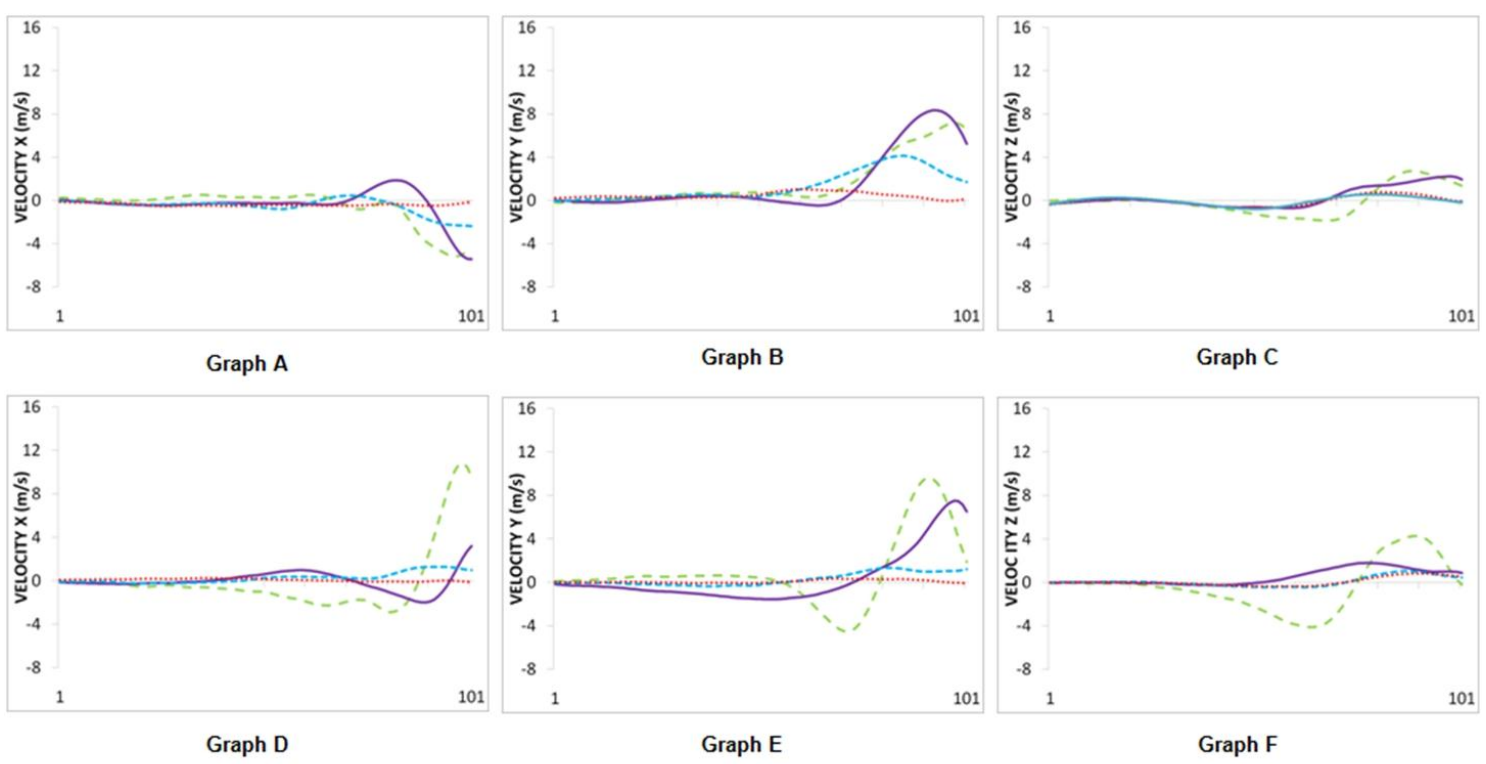

- -Wrist —Elbow ---Shoulder .....Hip

Figure 4. Typical Joint Component Velocity for Single maximal Cross and Lead Hook.

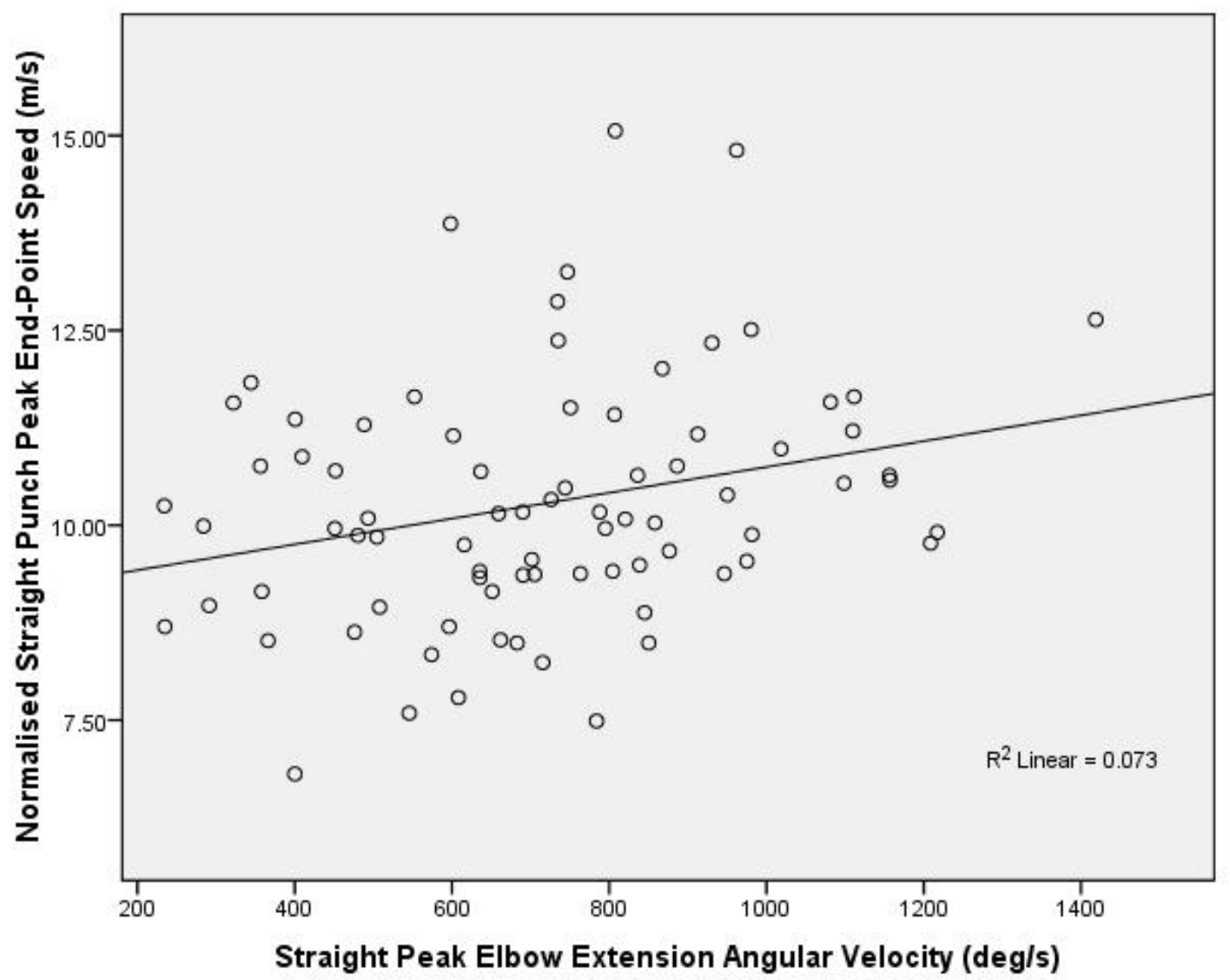

Page $\mathbf{2 1}$ of 22 
Figure 5. Relationship between normalised peak contact speed and peak elbow extension angular velocity for a Jab and Cross (i.e. Straight) punches. Each data point is the mean for each subject for each punch type and modality $(\mathrm{N}=80)$. 